\title{
BMJ Open Healthcare provision, functional ability and quality of life after proximal femoral fracture - 'ProFem': Study protocol of a population-based, prospective study based on individually linked survey and statutory health insurance data
}

To cite: Andrich S, Ritschel M, Meyer $\mathrm{G}$, et al. Healthcare provision, functional ability and quality of life after proximal femoral fracture - 'ProFem': Study protocol of a populationbased, prospective study based on individually linked survey and statutory health insurance data. BMJ Open 2019;9:e028144. doi:10.1136/ bmjopen-2018-028144

- Prepublication history and additional material for this paper are available online. To view these files, please visit the journal online (http://dx.doi. org/10.1136/bmjopen-2018028144).

SA and MR shared first authorship.

Received 28 November 2018 Revised 19 March 2019 Accepted 9 May 2019

\section{Check for updates}

(c) Author(s) (or their employer(s)) 2019. Re-use permitted under CC BY-NC. No commercial re-use. See rights and permissions. Published by BMJ.

For numbered affiliations see end of article.

\section{Correspondence to} Dr Silke Andrich; silke.andrich@uni-duesseldorf. de

Silke Andrich (D) , ${ }^{1,2}$ Michaela Ritschel, ${ }^{1}$ Gabriele Meyer, ${ }^{3}$ Falk Hoffmann, ${ }^{4}$ Astrid Stephan, ${ }^{3}$ Marion Baltes, ${ }^{3}$ Juliane Blessin, ${ }^{3}$ Kathrin Jobski, ${ }^{4}$ Alexander M Fassmer, ${ }^{4}$ Burkhard Haastert, ${ }^{1,5}$ Veronika Gontscharuk, ${ }^{1}$ Werner Arend, ${ }^{1}$ Lena Theunissen, ${ }^{1}$ Denise Colley, ${ }^{1}$ Raoul Hinze, ${ }^{1}$ Simon Thelen, ${ }^{6}$ Petra Fuhrmann, ${ }^{7}$ Christian G G Sorg, ${ }^{7}$ Joachim Windolf, ${ }^{6}$ Christoph J Rupprecht, ${ }^{7}$ Andrea lcks ${ }^{1,2}$

\section{ABSTRACT}

Introduction Proximal femoral fractures (PFF) are among the most frequent fractures in older people. However, the situation of people with a PFF after hospital discharge is poorly understood. Our aim is to (1) analyse healthcare provision, (2) examine clinical and patientreported outcomes (PROs), (3) describe clinical and sociodemographic predictors of these and (4) develop an algorithm to identify subgroups with poor outcomes and a potential need for more intensive healthcare.

Methods and analysis This is a population-based prospective study based on individually linked survey and statutory health insurance (SHI) data. All people aged minimum 60 years who have been continuously insured with the AOK Rheinland/Hamburg and experience a PFF within 1 year will be consecutively included (SHI data analysis). Additionally, 700 people selected randomly from the study population will be consecutively invited to participate in the survey. Questionnaire data will be collected in the participants' private surroundings at 3 , 6 and 12 months after hospital discharge. If the insured person considers themselves to be only partially or not at all able to take part in the survey, a proxy person will be interviewed where possible. SHI variables include healthcare provision, healthcare costs and clinical outcomes. Questionnaire variables include information on PROs, lifestyle characteristics and socioeconomic status. We will use multiple regression models to estimate healthcare processes and outcomes including mortality and cost, investigate predictors, perform non-responder analysis and develop an algorithm to identify vulnerable subgroups.

Ethics and dissemination The study was approved by the ethics committee of the Faculty of Medicine, Heinrich-

\section{Strengths and limitations of this study}

To the best of our knowledge, this is the first study to conduct an individual data linkage between statutory health insurance (SHI) data and questionnaire data in the field of research on proximal femoral fractures (PFF).

- Individually linked survey and SHI data are used to answer a variety of health service research, clinical and patient-orientated questions in people with PFF.

- Vulnerable subgroups, such as people with dementia, are included in our study.

- Due to structural differences between populations insured with various SHI funds and regions, the generalisability of our findings might be limited.

Heine-University Düsseldorf (approval reference 6128R). All participants including proxies providing written and informed consent can withdraw from the study at any time. The study findings will be disseminated through scientific journals and public information.

Trial registration number DRKS00012554.

\section{INTRODUCTION}

Proximal femoral fractures (PFF) are among the most frequent fractures in older people. ${ }^{12}$ However, knowledge about the situation of those affected by a PFF is scarce. ${ }^{3-5}$ Studies indicate poor outcomes following a PFF: $50 \%$ of those affected retained functional limitations, ${ }^{6} 15 \%$ were newly admitted to a nursing 
home $^{7}$ and around $20 \%$ died within 1 year. $^{8}$ Although postoperative programmes showed positive effects, ${ }^{6-11}$ more than $60 \%$ of patients received no further treatment, ${ }^{6}$ suggesting shortcomings in the care provided. Specific aspects of healthcare provision, such as treatment in geriatric trauma centres or rehabilitation, have hardly been investigated. Existing international studies suggest a healthcare gap. ${ }^{6}{ }^{12}$ It is currently unclear as to which patients particularly benefit from specific care models. ${ }^{13}$ In addition to healthcare processes, patient-reported outcomes (PROs) such as health-related quality of life (HRQoL), functional ability and social participation in older people following PFF have hardly been investigated. Subgroups characterised by particularly poor clinical and PROs, and by a potential need of more intensive care, have not yet been identified.

Therefore, the aims of this study are (1) to analyse healthcare provision after PFF, (2) to examine clinical outcomes (such as re-hospitalisation, occurrence of need for care, nursing home admission, death) and PROs (such as HRQoL, functional ability, social participation) after PFF and (3) to describe clinical and sociodemographic predictors of these (such as comorbidity, age, sex, social support). In doing so, (4) the aim is to identify subgroups who have poor outcomes (eg, people living at home with low social support, comorbidity and high healthcare utilisation) and are potentially in need of more intensive healthcare. This will be done by developing an algorithm which generates a 'case finding' to detect those groups of people.

Our project is funded by the Innovation Fund coordinated by the Innovation Committee of the Federal Joint Committee (grant number: 01VSF16043).

\section{METHODS AND ANALYSIS}

\section{Study design and population}

This is a population-based prospective study based on statutory health insurance (SHI) data and questionnaire data collected from people insured with the AOK Rheinland/Hamburg. Overall, the AOK Rheinland/Hamburg covers more than 2.5 million insured people in North Rhine-Westphalia (NRW), which has the highest population of all German Federal States, with approximately $25 \%$ aged 60 years or older. All people resident in NRW aged 60 years or above and who have been continuously insured with the AOK Rheinland/Hamburg for at least 12 months prior to PFF and experience a PFF between January 2018 and January 2019 will be consecutively included in the study. People with PFF will be identified consecutively over 1 year along with their exact date of hospital discharge using SHI diagnoses (main or secondary diagnosis) and operational procedure keys. A fracture event is defined according to the 10th revision of the International Classification of Diseases (ICD-10) codes S72.0 (fracture of head and neck of femur), S72.1 (pertrochanteric femoral fracture) and S72.2 (subtrochanteric femoral fracture), and selected surgical and procedural keys (OPS-Codes, see online supplementary appendix A).

This study comprises two populations: all identified people as described above belong to the (1) study population based on SHI data. For those, a comprehensive analysis of SHI data covering 12 months before and 12 months after the fracture event will be performed. Furthermore, a (2) random sample drawn from the overall SHI study population - will be consecutively invited to additionally participate in a survey. An algorithm will be applied weekly to ensure a random selection of the survey sample. Questionnaire data collection is planned at 3 and 12 months after hospital discharge using Pen-and-Paper Personal Interviews with participants in a private surrounding, and at 6 months after discharge by means of a postal survey. Sufficient German language skills are a prerequisite for participation in the survey (see online supplementary appendix B). If the insured person considers themselves to be only partially or not at all able to take part in the survey, for example, due to dementia or reduced state of health, an attempt will be made to conduct the interview with a caregiving relative (person of trust) or a legal guardian either additionally or on behalf of the insured person. The following criteria will be used to identify an eligible proxy: they must know the insured person well, should visit the insured person twice a week on average and support them in everyday life. The participation of the proxy is always voluntary. If a person is too ill to be interviewed at baseline but willing to stay in the study, we will try to arrange an interview at the next time interval. People no longer able to take part in the interviews themselves and with no eligible caregiving relative or legal guardian to perform a proxy interview with will be excluded (see online supplementary appendix B).

Figure 1 displays the flow through the study. The sample size calculation will be described further below.

\section{Recruitment}

First contact to arrange an appointment for the visit in the private surrounding will be made by postal letter. The letter contains a cover letter, information on the study and on data protection, and the consent form for participation for prior information. The letter will also ask the insured person (or their proxy) to contact the study centre to arrange an appointment. A written reminder will be sent to non-responders after approximately 2 weeks, followed by telephone contact as a next step. Where no telephone number is available, a second reminder will be sent. Response will be monitored consecutively and proportions will be calculated to describe participation behaviour. ${ }^{1415}$

\section{Data collection - data sources and variables}

Data will be collected from the sources outlined above. SHI data collected from consenting individuals 12 months before and 12 months after the event will be 
Identification of people with proximal femoral fracture (PFF) $(n=4000)$

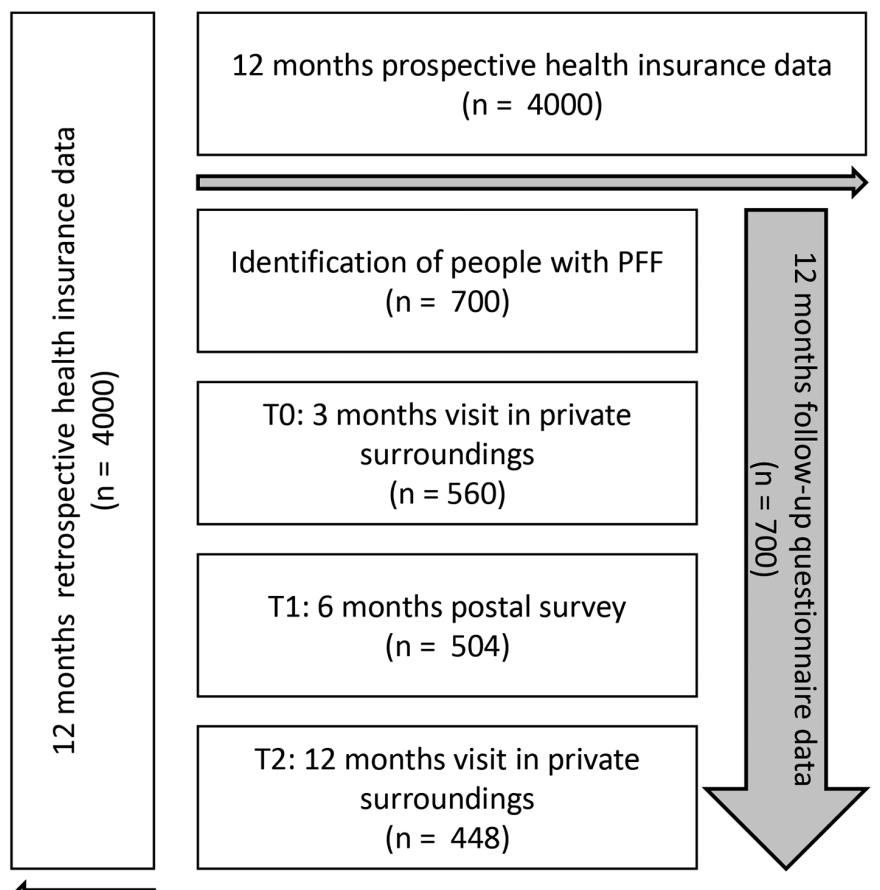

Figure 1 Study design.

individually linked to questionnaire data. The SHI data will be used to measure healthcare provision and clinical outcomes in the 12 months after PFF. Healthcare provision is described for various healthcare areas: inpatient and outpatient care, rehabilitation, nursing services, prescribed medication, remedies and medical aids as well as costs for the different healthcare areas, transportation, and costs in total. Clinical outcomes are re-hospitalisation, care dependency (including new occurrences), admission to a nursing home and mortality. SHI data collected 12 months after PFF and 12 months before PFF will also be evaluated regarding predictors such as demographic characteristics and comorbidity. The questionnaire data will be used to record PROs, which focus on HRQoL, functional ability, social participation, pain, fear of falling and falls. Self-reported predictors include demographic characteristics as well as socioeconomic status, social support, lifestyle, healthcare utilisation and special health-related events. Questions on PROs should be answered by the insured person whenever possible. If the insured person is not able to respond to an abridged version of the questionnaire comprising the questions on the aforementioned PROs, the proxy will be asked to respond to the entire questionnaire except the questions on pain and fear of falling (please see table 1). Interviews will be conducted by trained interviewers. An interviewer manual and standard operating procedures will be provided for quality assurance purposes and to ensure a standardised approach.

\section{Outcomes}

The following variables will be recorded for the aforementioned purpose:

\section{SHI data}

\section{Healthcare provision}

Healthcare provision will be evaluated using the following variables: number of hospital admissions after PFF; length of hospital stay (LOS) for each hospital admission; admission to a specialist department; physician contact per billing quarter; number and kind of different specialists involved; number, duration and kind of inpatient and/or outpatient rehabilitation; number, duration and kind of nursing services; institutional long-term care or short-term care; number and kind of prescribed medications; and number and kind of remedies and medical aids. Furthermore, healthcare costs will include the following: inpatient costs; outpatient costs; rehabilitation costs; nursing services costs; medication costs; costs for remedies and medical aids; and costs for transportation. Healthcare costs will be shown in euro. Since outpatient data are only provided in quarters, these cost values will be equally distributed over the time span (eg, in weeks).

\section{Clinical outcomes}

Re-hospitalisation is addressed by the variables already named above (number of hospital admissions after PFF, LOS for each hospital admission and admission to a specialist department). Care dependency (including new occurrences) is defined by a classification system for a person's impairment of autonomy and displayed in five care degrees according to the German Nursing Care Act. The five care degrees are depending on the amount of care needed and with a range from the level of care 1 (minor impairment of the person's autonomy) up to level 5 (heaviest impairment with special demands on nursing care) ${ }^{16}$ The maximum level of care in the period before the PFF hospital stay will be considered. Admission to a nursing home is assessed by the type of service, including, for example, provision of short-term or long-term care along with the exact date. The date of death of people with PFF who died during the observation period will be recorded to assess mortality.

\section{Questionnaire data}

Patient-reported outcomes

\section{Health-related quality of life}

Overall HRQoL will be measured using the 12-Item Short Form Health Survey (SF-12) covering the previous 4 weeks of a person's life. ${ }^{17}$ The SF-12 is the shorter version of the Short Form-36 Health Survey (SF-36) and contains one or two items for the following eight health dimensions: physical functioning, role functioning, bodily pain, general health perception, energy/vitality, social functioning, emotional functioning and mental health. The SF-12 comprises two summary scores - a physical component summary score (PCS) and a mental component summary score (MCS). In the SF-12, a better HRQoL is denoted 


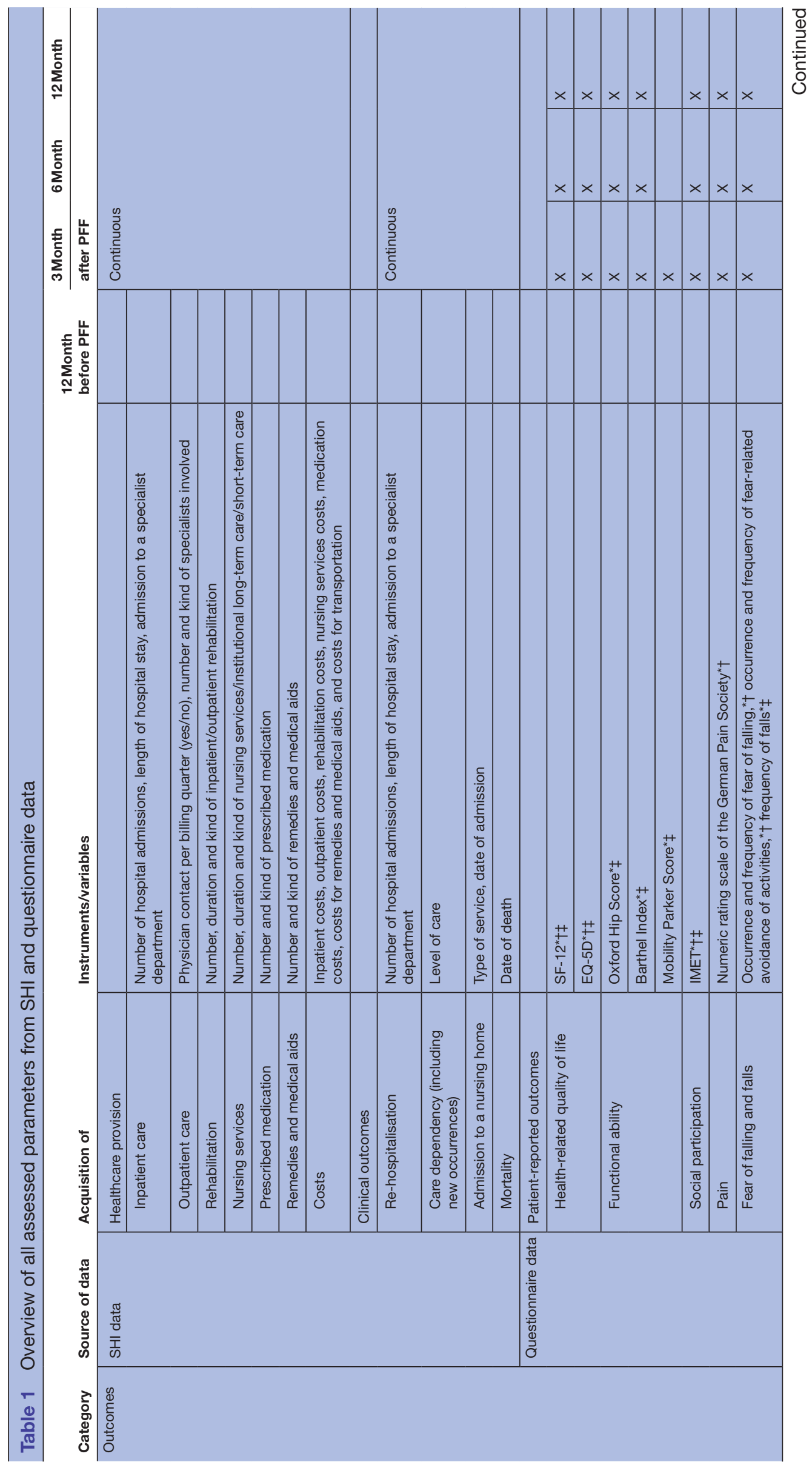

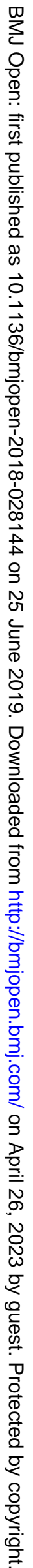




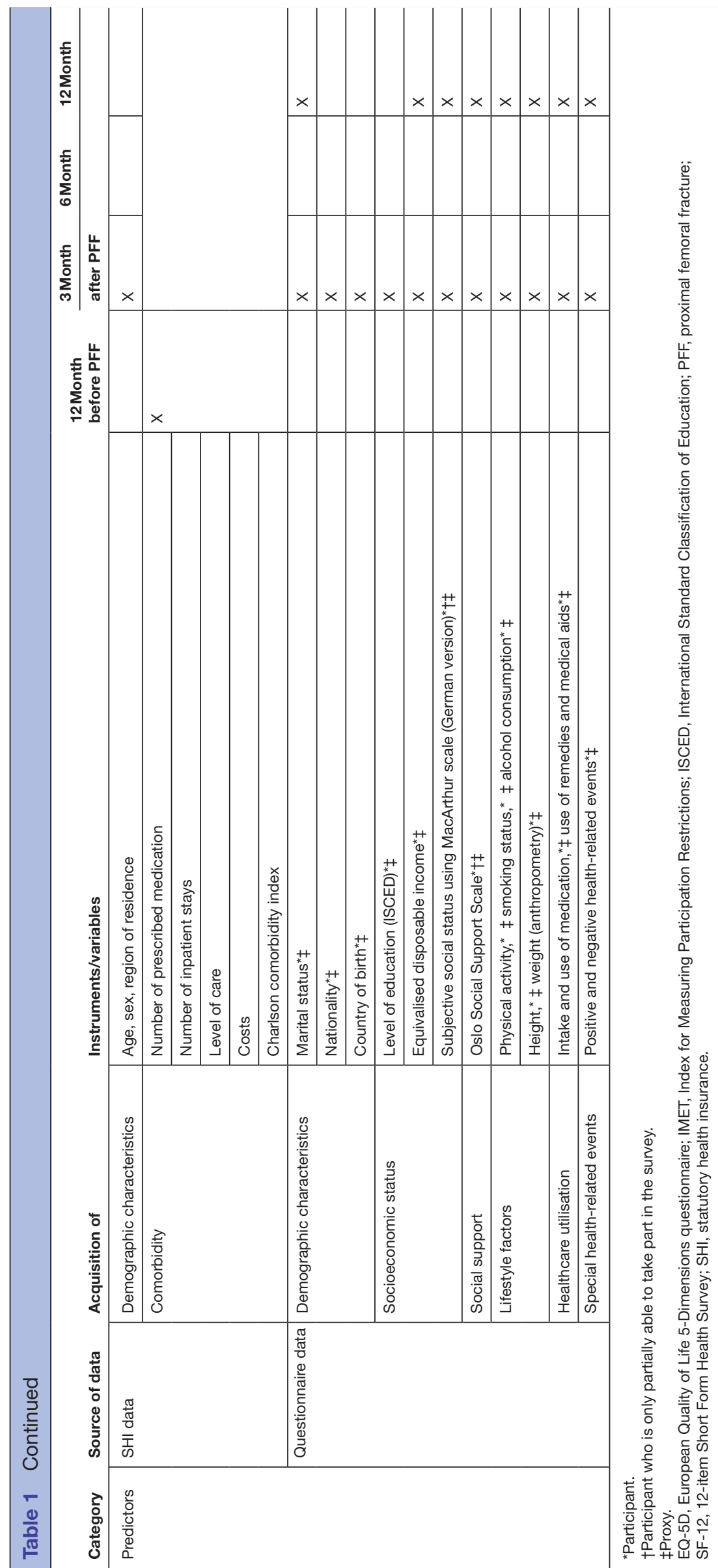

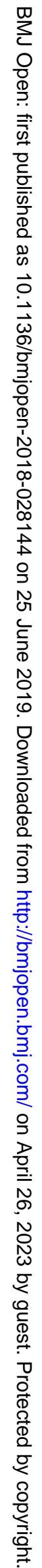


by higher values. The SF-12 is one of the best known and most frequently used instrument to assess HRQoL and its measurement performance has been tested. ${ }^{18}$

The European Quality of Life 5-Dimensions questionnaire (EQ-5D) is a generic preference-based measure of health status and consists of two parts - a questionnaire and a visual analogue scale (EQ-VAS). ${ }^{19} 20$ The questionnaire contains five dimensions: mobility, self-care, usual activities, pain/discomfort and anxiety/depression. Each dimension is rated on a five-level scale. The result of the questionnaire can be summarised as perceived state of health (from best to worst state where '11111' is the best and ' 55555 ' the worst state). The EQ-VAS is a scale for rating health between 100 (best imaginable state of health) and zero (worst imaginable state of health), expressing health on the day of completion. The EQ-5D is a well-accepted and frequently used instrument with good psychometric properties. ${ }^{21}$

\section{Functional ability}

Functional ability will be measured using three instruments.

The Oxford Hip Score (OHS) is a hip-specific 12-item questionnaire to assess activities of daily living (ADL) with six questions relating to pain (type and the resulting impairments in mobility) and six questions relating to functional ability (self-care, mobility and independence), each referring to the last 4 weeks. ${ }^{22}{ }^{23}$ Answers to the questions are categorised in five dimensions, ranging from 0 (worst outcome) to 4 (best outcome). The sum of all values provides an overall score between 0 and 48. A high overall score indicates a better outcome with 48 being the best outcome. The reliability and validity of the OHS have been assessed in a German population with osteoarthritis of the hip. ${ }^{24}$

The Heuschmann et $a l^{25}$ version of the Barthel Index (BI), a common measure of ADL, provides an interview and a paper and pencil form. The BI comprises 10 domains which are divided into self-care and mobility. Selfcare includes feeding, bathing, grooming, dressing, toilet use, and bowel and bladder control. Mobility consists of transfer, mobility and stairs. The interview version comprises 17 questions. The postal survey includes 10 questions - one for each domain. The person's overall ADL score is classified according to an ordinal scale where 0 is the lowest score and 20 indicates complete independence. Comprehensibility and reliability of both questionnaires were tested in patients with stroke. ${ }^{25}$

Pre-fracture functional level will be assessed using the Mobility Parker Score, ${ }^{26}$ which evaluates a person's ability to get about the house, out of the house and to go shopping. Each item is rated from 0 ('no difficulty') to 3 ('not at all'). The answers result in a total score ranging from 'no walking ability at all' to 'total independence' (0-9).

\section{Social participation}

The Index for Measuring Participation Restrictions (IMET) measures the restriction of participation of people with different chronic diseases. ${ }^{27} 28$ Based on the dimensions of the International Classification of Functioning, Disability and Health, this tool covers nine aspects of everyday activities and participation. This includes self-care, daily duties, and responsibilities at and outside home, recreation, social activities, personal relations, sex, and stress and extraordinary strains. The impairment caused by a disease is evaluated for each item by means of an 11-level scale (0-10). Lower IMET values suggest better social participation. A summary score can be calculated. The psychometric properties have been tested for different patient groups. ${ }^{28}$

Pain

Pain will be recorded as follows: the current level of pain, the average level of pain during the last 4 weeks and the highest level of pain during the last 4 weeks will be rated on a numerical rating scale $(1-5) \cdot{ }^{29}$

\section{Fear of falling and falls}

The assessment of fear of falling ${ }^{30}$ includes a question about occurrence and frequency of fear, ${ }^{31}$ and a question about the occurrence and frequency of activities avoided due to fear of falling. ${ }^{31}$ The number and frequency of falls are recorded over the 12 months prior to the fracture as well as the period after the fracture. ${ }^{32} 33$ A single question is used to determine whether it is the first fracture.

Overall, we considered the recent recommendations regarding core outcomes and appropriate instruments to be used in trials with older people with hip fractures. ${ }^{34-36}$ We selected instruments that are suitable for personal interviews, postal survey and proxy assessments. We decided for the SF-12 instead of the SF-36 to reduce the length of the questionnaire. ${ }^{34}$

\section{Predictors}

\section{SHI data}

\section{Demographic characteristics and comorbidity}

Age, sex and region of residence will be considered. Comorbidity, number of prescribed medications, number of inpatient stays in hospital, level of care and healthcare costs for the year prior to the fracture will be considered as possible predictors 12 months before PFF. Age will be defined by the year of the fracture event and classified into 5-year age groups. Region of residence will be denoted by the first two numbers of the postcode. Additionally, overall comorbidity-related disease burden will be assessed using the enhanced Charlson comorbidity index for ICD-10 codes. ${ }^{37} 38$ In accordance with previous studies, ${ }^{39-42}$ the Charlson comorbidity index will be calculated using inpatient diagnoses 52 weeks before $(<)$ the index week and outpatient diagnoses four quarters before $(<)$ the index quarter. A score variable will sum up and categorise comorbidities from $0,1,2-3,4-5$ and $6+$.

\section{Questionnaire data}

\section{Demographic characteristics}

The assessment of demographic characteristics comprises marital status, nationality and country of birth. 


\section{Socioeconomic status}

The socioeconomic status includes education and income. Education will be recorded based on the International Standard Classification of Education. Education level is grouped into three categories ranging from low to high. ${ }^{43}$ Income will be determined by the equivalised disposable income. For this purpose, the net household income will be recorded by providing 15 categories of income, the household size and the number of people living in the household including information regarding sex, age and relationship to the participant. ${ }^{33}$ The number of children, professional position, indication of professional activity and information on the current employment situation will also be assessed. Subjective social status will be rated using a 10-rung self-anchoring scale in the form of a ladder. $^{44} 45$

\section{Social support}

Social support will be captured using the Oslo Social Support Scale, which consists of three questions regarding close people to rely on; other people's concern and interest in the participant's life; and ease of obtaining help from neighbours. ${ }^{46}$ Answers will be scored from 1 to 4 or 5 . The individual values will be added to a total score, which can have values between 3 and 14 with higher values indicating higher social support. $^{47}$

\section{Lifestyle factors}

Questions regarding lifestyle factors include the following: physical activity $^{33} 48$; smoking status, and if applicable the amount of cigarettes, cigars, pipes and cigarillos smoked per day ${ }^{49} 50$; alcohol consumption and binge drinking. ${ }^{33}$ Height and weight will be recorded as anthropometric measures.

\section{Healthcare utilisation}

Intake and use of medication will be recorded within five questions to assess healthcare utilisation beyond SHI data. Participants will be asked about their current intake of analgesics, use of over the counter medication and the presence of a medication plan. Photographs will be taken of the plan or drug package(s) and a question asked about who is responsible for drug preparation. It will also be assessed if medications are administered long term or prescribed as needed. Furthermore, the current pattern of use of remedies and medical aids and their possession before the fracture will be recorded using different pictures of remedies and medical aids. ${ }^{51}$

\section{Special health-related events}

The assessment of special negative and positive health-related events as significant incidents comprises the previous 6-month period and the last 7 days. Participants will be asked if they experienced any positive or negative events and if so, which events can be named. ${ }^{52}$

Table 1 provides an overview of all assessed parameters from SHI and questionnaire data.

\section{Patient and public involvement}

Patients were not involved in the definition of the research questions and modelling of the design and outcome measures. They were also not engaged in the recruitment and conduct of the study. Our aim is to include patients in the interpretation of the study results if possible. Public involvement is achieved through the active role of the AOK Rheinland/Hamburg, a SHI company that represents the interests of its members. The results of the study will be disseminated to the study participants through public information such as the customer magazine $A O K$ Vigo.

\section{Sample size}

According to data from the year 2014 provided by the AOK Rheinland/Hamburg, at least 4000 insured people aged 60 years or above are expected to experience a PFF within 12 months. Of these, 700 randomly selected people will be consecutively invited to participate in the survey. The sample size should make it possible to show specific healthcare provision (rehabilitation, treatment and medical care), and outcomes in relevant subgroups (age, sex, migration background and various social contexts). The data currently available from literature are insufficient for performing a detailed calculation of sample size. However, age- and sex-specific estimates are available for HRQoL, ${ }^{53}$ mortality ${ }^{8}$ and rehabilitation. ${ }^{54}$ The precision of the estimates of these parameters has been examined a priori, assuming the expected 4000 people with PFF and the random sample of $n=700$ based on the age and sex distribution of the insured population of AOK Rheinland/Hamburg in 2014. The aim is to achieve a response of $80 \%$ of the target population. A dropout of $20 \%$ is expected after 12 months, including participants who die (approximately 20\%). ${ }^{8}$ This results in $448(=0.8 \times 0.8 \times 700)$ participants after 12 months. The precision of the estimators was evaluated by calculating the PCS and MCS for HRQoL based on the sample size stratified by age and sex. From the results presented in table 2, it can be concluded that the sample size provides sufficient accuracy for estimating HRQOL. It will be possible to identify differences between men and women and age groups. If the observed response considerably differs from the expected response, we will adjust the number of weekly contacted persons up to exhausting the whole SHI study population.

\section{Planned statistical analysis}

Depending on the research question, statistical analyses are carried out using either (1) SHI data or (2) SHI data and questionnaire data, which are combined to a linkage dataset. In general, the SHI population of all identified patients with PFF and the survey sample will be described using baseline variables by prevalence (with $95 \% \mathrm{CI}$ ), means, medians, SD or percentiles depending on their distributions.

The variables of healthcare provision will be investigated using descriptive methods. Furthermore, healthcare provision will be evaluated by latent class analysis (LCA) and latent transition analysis (LTA). LCA is a modelling technique used to categorise participants into 
Table 2 Precision of the estimators of the physical and mental component summary score (SF-36)

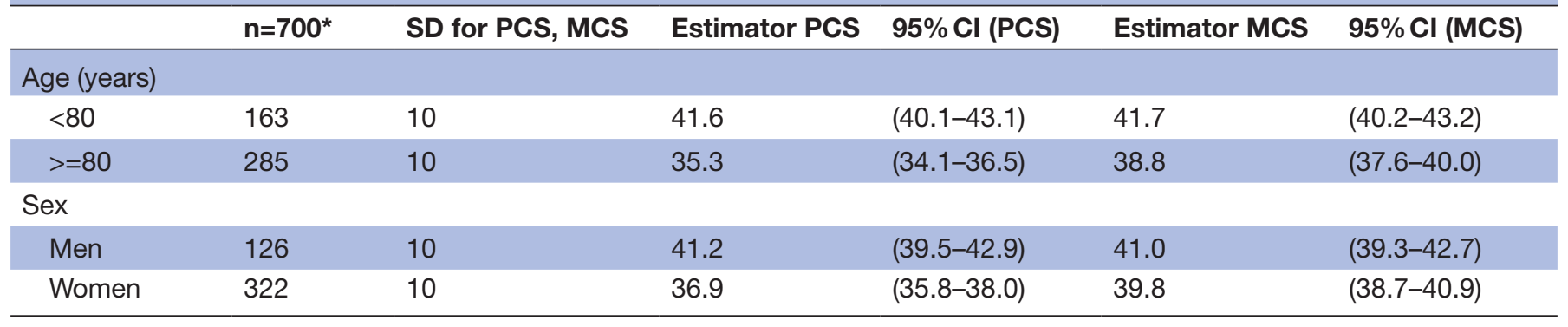

${ }^{*} \mathrm{n}=448$ after 12 months.

MCS, mental component summary score; PCS, physical component summary score; SF-36, Short Form-36 Health Survey.

a number of unique (unobserved) classes. Participants are homogeneous with respect to their healthcare utilisation within each latent class and heterogeneous between classes. LCA typically uses cross-sectional data to identify subgroups at a single time point. LTA is an extension of LCA using longitudinal data where individuals transition between latent classes over time. ${ }^{55}$ Healthcare costs will be displayed in euro and categorised in approximately quintile classes. Calculations of total mean costs and mean costs per component and a $95 \%$ bootstrap CI will be performed.

Clinical outcomes will be analysed using Kaplan-Meier survival curves and Cox regression. Two analyses will be conducted regarding mortality, the first for the entire SHI study population, and the second for the survey sample addressing the prognosis for people who have already survived at least 3 months. The mortality of the SHI study population will be compared with the German population $\geq 60$ years. Age- and sex-specific relative mortality rates will be calculated. Standardised mortality ratios and comparative mortality figures will be estimated together with $95 \% \mathrm{CI}$.

PROs will be studied using stratified descriptive analysis. Among others, outcomes related to PROs will be considered separately according to dementia status and state of health. Graphical or regression methods will be used to describe and exploratively estimate the association between paired self-reported and proxy values in the subpopulation of participants with dementia/ reduced state of health at fixed time points. It will be discussed, whether imputation of transformed proxy values in missing outcome values should be done. Further subpopulations will be considered for sensitivity analyses: participants without dementia/reducedstate of health, participants with dementia/reduced state of health only with self-reported values, respectively, only with proxy values. Furthermore, participants changing between self-reported and proxy values during follow-up will be described separately. Depending on frequencies and results, specific imputation methods for self-reported values will be discussed.

Possible predictors to healthcare provision, clinical and PROs will be investigated using mixed linear or logistic regression models, depending on the distribution of outcomes. Furthermore, two-part models ${ }^{56-58}$ will be used to investigate associations within cost analysis. Repeated measurements per participant will be adjusted by random effects in the mixed models.

At least one binary indicator for 'severe cases' will be derived from the outcomes. Different competing definitions for 'severe cases' could be combined in order to choose the final indicator. Furthermore, a LCA or LTA including different variables might be considered. An algorithm will be derived from one half of the study population based on the binary case indicator using logistic regression models with independent variables selected from the baseline variables to classify the risk of a 'severe case' after PFF. A final selection of variables for the algorithm will be made using goodness of fit criteria and stepwise, forward and backward selection procedures. A score will be derived via the final model and tested with the other half of the study population (randomly chosen).

Furthermore, a non-responder analysis will be performed based on individual SHI data, available for responders and non-responders. Descriptive statistics and corresponding statistical tests will be used to describe response at all observation intervals. Logistic regression analysis with subsequent multiple adjustment will be used to obtain (adjusted) ORs for belonging to the response group.

The study and the data linkage will be performed in line with Good Epidemiological Practice (GEP), ${ }^{59}$ Good Practice of Secondary Data Analysis (GPS), ${ }^{60}$ and in accordance with the REporting of studies Conducted Using Observational Routinely-Collected Health Data Statement, ${ }^{61}$ and the Standard for Secondary Data Analyses. $^{62}$ The data linkage of questionnaire and SHI data will be performed on an individual level using suitable key variables.

\section{DISCUSSION AND PRACTICAL IMPLICATIONS}

The results of the study may contribute to improving older people's HRQoL, functional ability and social participation as well as to the reduction of costs associated with the avoidable need of care and hospitalisation after PFF. 


\section{CONCLUSION}

To our knowledge, this is the first study to investigate questions regarding healthcare provision, HRQoL, functional ability and social participation after PFF. The strength of our study is the linkage of SHI and questionnaire data as well as the consideration of important vulnerable subgroups, such as people with dementia.

\section{ETHICS AND DISSEMINATION}

The study was approved by the responsible ethics committee of the Faculty of Medicine, Heinrich-Heine-University Düsseldorf (approval reference 6128R). All participants will provide written and informed consent and can withdraw from the study at any time. All procedures performed will be in accordance with the Declaration of Helsinki and comparable ethical standards (eg, GEP and GPS). The data protection agreement applied for this study does not cover posting data in public databases. Data will be held at the Institute for Health Services Research and Health Economics and mediStatistica. The development of this study protocol was guided by the Standard Protocol Items: Recommendations for Interventional Trials (SPIRIT) statement and the SPIRIT-PRO Extension, where applicable. ${ }^{63-65}$ The results of the project will help to identify possible shortcomings in the care of older people with PFF and detect people with special needs of care. The findings of the study will be disseminated through scientific journals and public information.

\section{Author affiliations}

${ }^{1}$ Institute for Health Services Research and Health Economics, Centre for Health and Society, Faculty of Medicine, Heinrich-Heine-University Düsseldorf, Düsseldorf, Germany

${ }^{2}$ Institute for Health Services Research and Health Economics, German Diabetes Center, Leibniz Center for Diabetes Research at the Heinrich-Heine-University Düsseldorf, Düsseldorf, Germany

${ }^{3}$ Martin Luther University Halle-Wittenberg, Institute for Health and Nursing Sciences, Medical Faculty, Halle (Saale), Germany

${ }^{4}$ Department of Health Services Research, Faculty of Medicine and Health Sciences, Carl von Ossietzky University Oldenburg, Germany

${ }^{5}$ mediStatistica, Neuenrade, Germany

${ }^{6}$ Department of Trauma and Hand Surgery, University Hospital Düsseldorf, Germany ${ }^{7}$ Department Health Policy - Health Economics - Press Relations, AOK Rheinland/ Hamburg, Düsseldorf, Germany

\section{Correction notice This article has been corrected since it was published.}

Acknowledgements We thank all participants and their caregiving relatives (person of trust) or legal guardians for the support of our study.

Contributors SA, MR and Al drafted the manuscript. SA, MR, GM, FH, AS, MB, JB, $\mathrm{KJ}, \mathrm{AF}, \mathrm{BH}, \mathrm{VG}, \mathrm{WA}, \mathrm{LT}, \mathrm{DC}, \mathrm{RH}, \mathrm{ST}, \mathrm{PF}, \mathrm{CGGS}, \mathrm{JW}$, and CJR participated in the design and preparation of the study. BH and VG provided statistical analysis support. GM, $\mathrm{FH}, \mathrm{AS}, \mathrm{MB}, \mathrm{JB}, \mathrm{KJ}, \mathrm{AF}, \mathrm{BH}, \mathrm{VG}, \mathrm{WA}, \mathrm{LT}, \mathrm{DC}, \mathrm{RH}, \mathrm{ST}, \mathrm{PF}, \mathrm{CGGS}$, JW, and CJR critically revised the manuscript's drafts. All authors approved the final version of the manuscript.

Funding This work was supported by the Innovation Fund coordinated by the Innovation Committee of the Federal Joint Committee (G-BA) in Germany; grant number: 01VSF16043.

Competing interests None declared.

Patient consent for publication Not required.
Provenance and peer review Not commissioned; externally peer reviewed.

Open access This is an open access article distributed in accordance with the Creative Commons Attribution Non Commercial (CC BY-NC 4.0) license, which permits others to distribute, remix, adapt, build upon this work non-commercially, and license their derivative works on different terms, provided the original work is properly cited, appropriate credit is given, any changes made indicated, and the use is non-commercial. See: http://creativecommons.org/licenses/by-nc/4.0/.

ORCID iD

Silke Andrich http://orcid.org/0000-0001-8884-7465

\section{REFERENCES}

1 Ballane G, Cauley JA, Luckey MM, et al. Secular trends in hip fractures worldwide: opposing trends East versus West. J Bone Miner Res 2014;29:1745-55.

2 Icks A, Arend W, Becker C, et al. Incidence of hip fractures in Germany, 1995-2010. Arch Osteoporos 2013;8:140.

3 Griffin XL, Parsons N, Achten J, et al. Recovery of health-related quality of life in a United Kingdom hip fracture population. The Warwick Hip Trauma Evaluation-a prospective cohort study. Bone Joint J 2015;97-B:372-82.

4 Martín-Martín LM, Arroyo-Morales M, Sánchez-Cruz JJ, et al. Factors Influencing Performance-Oriented Mobility After Hip Fracture. J Aging Health 2015;27:827-42.

5 Sletvold O, Helbostad JL, Thingstad P, et al. Effect of in-hospital comprehensive geriatric assessment (CGA) in older people with hip fracture. The protocol of the Trondheim Hip Fracture trial. BMC Geriatr 2011;11:18.

6 Stenvall M, Olofsson B, Nyberg L, et al. Improved performance in activities of daily living and mobility after a multidisciplinary postoperative rehabilitation in older people with femoral neck fracture: a randomized controlled trial with 1-year follow-up. $J$ Rehabil Med 2007;39:232-8.

7 Ohmann C, Smektala R, Pientka L, et al. [A New Model of Comprehensive Data Linkage - Evaluation of its Application in Femoral Neck Fracture]. Z. ärztl. Fortbild. Qual. Gesundh.wes 2005;99:547-54.

8 Smektala R, Ohmann C, Paech S, et al. [On the prognosis of hip fractures. Assessment of mortality after hip fractures by analyzing longitudinal data from acute and rehabilitative care]. Unfallchirurg 2005;108:927-37.

9 Cameron ID, Handoll HHG, Finnegan TP, et al. Co-ordinated multidisciplinary approaches for inpatient rehabilitation of older patients with proximal femoral fractures. Cochrane Database Syst Rev 2001;3:CD000106.

10 Handoll HHG, Cameron ID, Mak JC, et al. Multidisciplinary rehabilitation for older people with hip fractures. Cochrane Database Syst Rev 2009;4:CD007125.

11 Lögters T, Hakimi M, Linhart W, et al. [Early interdisciplinary geriatric rehabilitation after hip fracture : Effective concept or just transfer of costs?]. Unfallchirurg 2008;111:719-26.

12 Morrison RS, Siu AL. A comparison of pain and its treatment in advanced dementia and cognitively intact patients with hip fracture. J Pain Symptom Manage 2000;19:240-8.

13 Pioli G, Giusti A, Barone A. Orthogeriatric care for the elderly with hip fractures: where are we? Aging Clin Exp Res 2008;20:113-22.

14 Slattery ML, Edwards SL, Caan BJ, et al. Response rates among control subjects in case-control studies. Ann Epidemiol 1995;5:245-9.

15 Stang A, Moebus S, Dragano N, et al. Baseline recruitment and analyses of nonresponse of the Heinz Nixdorf Recall Study: identifiability of phone numbers as the major determinant of response. Eur J Epidemiol 2005;20:489-96.

16 Kimmel A, Pflegereform BK. [Reform of the German Long-Term Care Insurance. A New Definition of Care Dependency and a New System to Assess the Need for Long-Term Care]. Gesundheitswesen 2016;78:477-88.

17 Ware J, Kosinski M, Keller SD. A 12-Item Short-Form Health Survey: construction of scales and preliminary tests of reliability and validity. Med Care 1996;34:220-33.

18 Morfeld M, Kirchberger I, Bullinger M. SF-36 - Fragebogen zum Gesundheitszustand. Deutsche Version des Short Form-36 Health Survey. 2. ergänzte und überarbeitete Auflage. Hogrefe Verlag, Göttingen, 2011.

19 Brooks R. EuroQol: the current state of play. Health Policy 1996;37:53-72. 
20 Herdman M, Gudex C, Lloyd A, et al. Development and preliminary testing of the new five-level version of EQ-5D (EQ-5D-5L). Qual Life Res 2011;20:1727-36.

21 Németh G. Health related quality of life outcome instruments. Eur Spine J 2006;15(Suppl 1):S44-S51.

22 Dawson J, Fitzpatrick R, Carr A, et al. Questionnaire on the perceptions of patients about total hip replacement. J Bone Joint Surg Br 1996;78:185-90.

23 Dawson J, Fitzpatrick R, Churchman D, et al. User Manual for the Oxford Hip Score (OHS). Version 1.0, August 2010: Oxford University Innovation Limited.

24 Naal FD, Sieverding M, Impellizzeri FM, et al. Reliability and validity of the cross-culturally adapted German Oxford hip score. Clin Orthop Relat Res 2009;467:952-7.

25 Heuschmann PU, Kolominsky-Rabas PL, Nolte $\mathrm{CH}$, et al. [The reliability of the german version of the barthel-index and the development of a postal and telephone version for the application on stroke patients]. Fortschr Neurol Psychiatr 2005;73:74-82.

26 Parker MJ, Palmer CR. A new mobility score for predicting mortality after hip fracture. J Bone Joint Surg Br 1993;75:797-8.

27 Deck R, Borowski C, Mittag O, et al. Index zur Messung von Einschränkungen der Teilhabe - Erste Ergebnisse eines ICForientierten Assessmentinstruments. DRV-Schriften 2006;64:152-3.

28 Deck R, Mittag O, Hüppe A, et al. Index zur Messung von Einschränkungen der Teilhabe (IMET) - Erste Ergebnisse eines ICF-orientierten Assessmentinstruments. Praxis Klinische Verhaltensmedizin und Rehabilitation 2007;76:113-20.

29 Deutsche Schmerzgesellschaft e.V. Deutscher Schmerz-Fragebogen Version 2015.2. Available: http://www.dgss.org/schmerzfragebogen/ [Accessed 15 Aug 2017].

30 Kempen GI, van Haastregt JC, McKee KJ, et al. Socio-demographic, health-related and psychosocial correlates of fear of falling and avoidance of activity in community-living older persons who avoid activity due to fear of falling. BMC Public Health 2009;9:170.

31 Zijlstra GA, van Haastregt JC, van Eijk JT, et al. Prevalence and correlates of fear of falling, and associated avoidance of activity in the general population of community-living older people. Age Ageing 2007;36:304-9.

32 Rapp K, Freiberger E, Todd C, et al. Fall incidence in Germany: results of two population-based studies, and comparison of retrospective and prospective falls data collection methods. BMC Geriatr 2014;14:105.

33 Robert Koch Institut. Gesundheitsfragebogen ab 65 Jahre (Studie zur Gesundheit Erwachsener in Deutschland - DEGS). März. 2009.

34 Haywood KL, Griffin XL, Achten J, et al. Developing a core outcome set for hip fracture trials. Bone Joint J 2014;96-B:1016-23.

35 Haywood KL, Brett J, Tutton E, et al. Patient-reported outcome measures in older people with hip fracture: a systematic review of quality and acceptability. Qual Life Res 2017;26:799-812.

36 Liem IS, Kammerlander C, Suhm N, et al. Identifying a standard set of outcome parameters for the evaluation of orthogeriatric comanagement for hip fractures. Injury 2013;44:1403-12.

37 Quan H, Sundararajan V, Halfon P, et al. Coding algorithms for defining comorbidities in ICD-9-CM and ICD-10 administrative data. Med Care 2005;43:1130-9.

38 Sundararajan V, Henderson T, Perry C, et al. New ICD-10 version of the Charlson comorbidity index predicted in-hospital mortality. J Clin Epidemiol 2004:57:1288-94.

39 Toson B, Harvey LA, Close JC. The ICD-10 Charlson Comorbidity Index predicted mortality but not resource utilization following hip fracture. J Clin Epidemiol 2015;68:44-51.

40 Klabunde CN, Potosky AL, Legler JM, et al. Development of a comorbidity index using physician claims data. J Clin Epidemiol 2000;53:1258-67.

41 Reyes C, Estrada P, Nogués X, et al. The impact of common comorbidities (as measured using the Charlson index) on hip fracture risk in elderly men: a population-based cohort study. Osteoporos Int 2014;25:1751-8.

42 Lix LM, Quail J, Teare G, et al. Performance of comorbidity measures for predicting outcomes in population-based osteoporosis cohorts. Osteoporos Int 2011;22:2633-43.

43 Avendano M, Jürges H, Mackenbach JP. Educational level and changes in health across Europe: longitudinal results from SHARE. $J$ Eur Soc Policy 2009;19:301-16.
44 Singh-Manoux A, Adler NE, Marmot MG. Subjective social status: its determinants and its association with measures of ill-health in the Whitehall II study. Soc Sci Med 2003;56:1321-33.

45 Hoebel J, Mueters S, Kuntz B, et al. [Measuring subjective social status in health research with a German version of the MacArthurs Scale]. Bundesgesundheitsb/ 2015;58:749-57

46 Kocalevent R, Brähler E. OSSS - Oslo Social Support Scale. In: Kemper C, Brähler E, Zenger M. Psychologische und sozialwissenschaftliche Kurzskalen. 1st edition. Berlin: Medizinisch Wissenschaftliche Verlagsgesellschaft mbH \& Co. KG, 2014:216-9.

47 Van Lente E, Barry MM, Molcho M, et al. Measuring population mental health and social well-being. Int $J$ Public Health 2012:57:421-30.

48 Lampert T, Mensink GBM, Müters S. [Physical and sporting activity among adults in Germany. Results from the "German Health Update 2009" survey]. Bundesgesundheitsb/ 2012;55:102-10.

49 Jöckel KH, Lehmann N, Jaeger BR, et al. Smoking cessation and subclinical atherosclerosis-results from the Heinz Nixdorf Recall Study. Atherosclerosis 2009;203:221-7.

50 Latza U, Hoffmann W, Terschüren C, et al. Quantifizierung und Analyse der Rauchexposition in epidemiologischen Studien. Berlin, Robert Koch-Institut 2005.

51 GKV-Hilfsmittelverzeichnis. Available: https://www.rehadat-gkv.de/ informationen/index.html [Accessed 15 Aug 2018].

52 Siegrist J, Broer M, Junge A. Profil der Lebensqualität chronisch Kranker. Manual. Göttingen: Beltz Test GmbH, 1996.

53 Moerman S, Vochteloo AJ, Tuinebreijer WE, et al. Factors associated with the course of health-related quality of life after a hip fracture. Arch Orthop Trauma Surg 2016;136:935-43.

54 Samuelsson B, Hedström MI, Ponzer S, et al. Gender differences and cognitive aspects on functional outcome after hip fracture-a 2 years follow-up of 2,134 patients. Age Ageing 2009;38:686-92.

55 Collins LM, Lanza ST. Latent Class and Latent Transition Analysis: With Applications in the Social, Behavioral, and Health Sciences. 2009:ISBN: 978-0-470-22839-5

56 Buntin MB, Zaslavsky AM. Too much ado about two-part models and transformation? Comparing methods of modeling Medicare expenditures. J Health Econ 2004;23:525-42.

57 Zou G. A modified poisson regression approach to prospective studies with binary data. Am J Epidemiol 2004;159:702-6.

58 Barber J, Thompson S. Multiple regression of cost data: use of generalised linear models. J Health Serv Res Policy 2004;9:197-204.

59 Guidelines and Recommendations to Assure Good Epidemiologic Practice (GEP), Long Version. German Society for Epidemiology (DGEpi). In Collaboration with the German Association for Medical Informatics, Biometrics, and Epidemiology (GMDS), German Association for Social Medicine and Prevention (DGSMP), German Region of the International Biometrics Association (DR-IBS). With revisions after evaluation April 2004. With supplement by implementation rule for Good Practice Secondary Data Analysis (GPS). 2008. Available: http://dgepi.de/fileadmin/pdf/GEP_LL_ english_f.pdf [Accessed 17 Feb 2018].

60 Swart E, Gothe H, Geyer S, et al. German Society for Social Medicine and Prevention; German Society for Epidemiology. [Good Practice of Secondary Data Analysis (GPS): Guidelines and Recommendations. Third Revision 2012/2014]. Gesundheitswesen 2015;77:120-6

61 Nicholls SG, Quach P, von Elm E, et al. The REporting of Studies Conducted Using Observational Routinely-Collected Health Data (RECORD) Statement: Methods for Arriving at Consensus and Developing Reporting Guidelines. PLoS One 2015;10:e0125620.

62 Swart E, Bitzer EM, Gothe H, et al. [A Consensus German Reporting Standard for Secondary Data Analyses, Version 2 (STROSASTandardisierte BerichtsROutine für SekundärdatenAnalysen)]. Gesundheitswesen 2016;78(S 01):e145-e160.

63 Chan AW, Tetzlaff JM, Altman DG, et al. SPIRIT 2013 statement: defining standard protocol items for clinical trials. Ann Intern Med 2013;158:200-7.

64 Chan AW, Tetzlaff JM, Gøtzsche PC, et al. SPIRIT 2013 explanation and elaboration: guidance for protocols of clinical trials. BMJ 2013;346:e7586.

65 Calvert M, Kyte D, Mercieca-Bebber R, et al. Guidelines for Inclusion of Patient-Reported Outcomes in Clinical Trial Protocols: The SPIRITPRO Extension. JAMA 2018;319:483-94. 